\title{
Ausbildungsinstitut für Klinische Verhaltenstherapie e.V.
} (AFKV)

Im laufenden Jahr werden im anerk. Institut weitere Kurse neu beginnen:

September 1992 VT-Ausbildung für Ärzte und Diplompsychologen

im Mai 1992 Zusatzausbildung in Kinder- und Jugendlichenverhaltenstherapie (in Kooperati-on mit der Abt. Klinische Psychologie der Universität Bremen).

Bei entsprechender Nachfrage ist daran gedacht, im Laufe des Jahres einen weiteren Kurs in Bremen zu beginnen (vgl. auch Verhaltenstherapie Heft 1, 1992).

An Sonderveranstaltungen ist ein Workshop mit Prof. F. Kanfer am 13./14.6.92 vorgesehen. Institutspolitisch hat das AFKV ein starkes Interesse an der sich anbahnenden gemeinsamen Haltung des FKV und der AVT gegenüber Organisationen und Institutionen, welche mit den Ausbildungskriterien und der Qualitätssicherung psychotherapeutischer Behandlung befaßt sind. Im Zuge des zu erwartenden Berufszulassungsgesetzes für heilkundlich tätige Diplompsychologen sind die durch die geltenden Psychotherapierichtlinien vorgegebenen Normen auch im Aus-bildungsbereich zu erhalten.

Um dies zu erreichen, müssen sich die Fachverbände der Verhaltenstherapie entsprechend gemeinsam einsetzen. Das AFKV Gelsenkirchen versucht darüber hinaus auch auf Landesebene eine größtmögliche Gemeinsamkeit mit den bestehenden Instituten in Nordrhein-Westfalen zu erreichen. Dies wird im Hinblick auf eine mögliche «Psychotherapeutenkammer» in diesem Land ein Mitspracherecht sichern.

Kontaktadresse:

\section{AFKV}

Cranger Straße 129

W-4650 Gelsenkirchen (BRD)

Tel. 0209/76490

Fax 02 09/78 0499 